Cite this: Phys. Chem. Chem. Phys., 2014, 16, 14083

Received 14th March 2014, Accepted 22nd May 2014 DOI: $10.1039 / c 4 c p 01106 f$

www.rsc.org/pccp

\title{
C K-edge NEXAFS spectra of graphene with physical and chemical defects: a study based on density functional theory $\dagger$
}

\author{
Christopher Ehlert, ${ }^{\mathrm{ab}}$ Wolfgang E. S. Unger*a and Peter Saalfrank ${ }^{\star b}$
}

\begin{abstract}
Recently, C K-edge Near Edge X-ray Absorption Fine Structure (NEXAFS) spectra of graphite (HOPG) surfaces have been measured for the pristine material, and for HOPG treated with either bromine or krypton plasmas (Lippitz et al., Surf. Sci., 2013, 611, L1). Changes of the NEXAFS spectra characteristic for physical (krypton) and/or chemical/physical modifications of the surface (bromine) upon plasma treatment were observed. Their molecular origin, however, remained elusive. In this work we study by density functional theory, the effects of selected point and line defects as well as chemical modifications on NEXAFS carbon $\mathrm{K}$-edge spectra of single graphene layers. For Br-treated surfaces, also $\mathrm{Br} 3 \mathrm{~d}$ X-ray Photoelectron Spectra (XPS) are simulated by a cluster approach, to identify possible chemical modifications. We observe that some of the defects related to plasma treatment lead to characteristic changes of NEXAFS spectra, similar to those in experiment. Theory provides possible microscopic origins for these changes.
\end{abstract}

\section{Introduction}

Graphene is a single layer of graphite, first manufactured and investigated in detail in 2004 by Novoselov, Geim and coworkers. ${ }^{1}$ This quasi two-dimensional material features a plethora of interesting electronic properties, ${ }^{2,3}$ which are promising for future applications, e.g. , in nano-electronics. ${ }^{4}$

One route towards applications is the functionalization of graphitic surfaces or graphene layers. ${ }^{5}$ A promising strategy is halogenation, e.g., bromination, since brominated HOPG (Highly Ordered Pyrolytic Graphite) surfaces are chemically reactive and versatile precursors to obtain alcohol or amine functional groups. The latter in turn serve to bind covalently, organic molecules for specific applications (see ref. 6 and references therein).

Recently, Lippitz et $a .^{6}$ reported on a bromine plasma treatment of graphene-like HOPG. It was found that such treatment leads to modifications in the $\mathrm{C}$ K-edge NEXAFS spectra compared to pristine HOPG. Because NEXAFS is a reliable, surface-sensitive tool for investigations of the electronic structure of materials, NEXAFS spectra are valuable tools to also unravel surface modifications due to functionalization.

\footnotetext{
${ }^{a}$ BAM Bundesanstalt für Materialforschung und -prüfung, D-12203 Berlin, Germany. E-mail: wolfgang.unger@bam.de

${ }^{b}$ Universität Potsdam, Institut für Chemie, Karl-Liebknecht-Str. 24-25, 14476 Potsdam-Golm, Germany. E-mail: petsaal@uni-potsdam.de

$\dagger$ Electronic supplementary information (ESI) available. See DOI: 10.1039/ c4cp01106f
}

C K-edge NEXAFS spectra for pristine and Br-plasma treated HOPG surfaces of ref. 6 are reproduced in Fig. 1(a). The spectra arise from transitions from the $\mathrm{C} 1 \mathrm{~s}$ orbital to various empty, bound final states. At the low-energy side, the NEXAFS spectrum of pristine HOPG shows a sharp resonance at a photon energy of $285.4 \mathrm{eV}$, corresponding to a $\mathrm{C} 1 \mathrm{~s} \rightarrow \pi^{*}$ transition. A second dominant feature is the double-structured resonance around $292 \mathrm{eV}$, corresponding to $\mathrm{C} 1 \mathrm{~s} \rightarrow \sigma^{*}$. This doubleresonance arises from excitonic (the sharp resonance at $291.8 \mathrm{eV}$ ) and band-like contributions (the broader signal at around $293 \mathrm{eV}$ ), according to ref. 8. Here, $\pi^{*}$ and $\sigma^{*}$ refer to antibonding molecular orbitals (more precisely: bands) of $\pi$ and $\sigma$ symmetry, respectively.

Upon bromination, the NEXAFS spectra change. The bromination with $\mathrm{Br}_{2}$ plasma in experiment was realized to different degrees, measured by $\mathrm{Br}$ atom percentages found by $\mathrm{Br} 3 \mathrm{~d}$ XPS in ref. 6. In Fig. 1(a), different curves refer to different $\mathrm{Br}$ percentages. Specifically, the following changes were observed upon $\mathrm{Br}_{2}$ treatment:

- The $\pi^{*}$ signal decreases with increasing $\mathrm{Br}$ content. Simultaneously, the $\sigma^{*}$ resonance increases, however, to a lesser extent.

- New resonances arise between the $\pi^{*}$ and the $\sigma^{*}$ resonance, in the energy range between $286 \mathrm{eV}$ and $290 \mathrm{eV}$. We shall sometimes call this region the "fingerprint" region in what follows.

- As a minor finding at very high $\mathrm{Br}$ concentrations, one observes a splitting of the $\pi^{*}$ resonance.

At this stage, the precise origin of these modifications is not known. In principle, Br plasma treatment can lead to physical 
(a) Br plasma

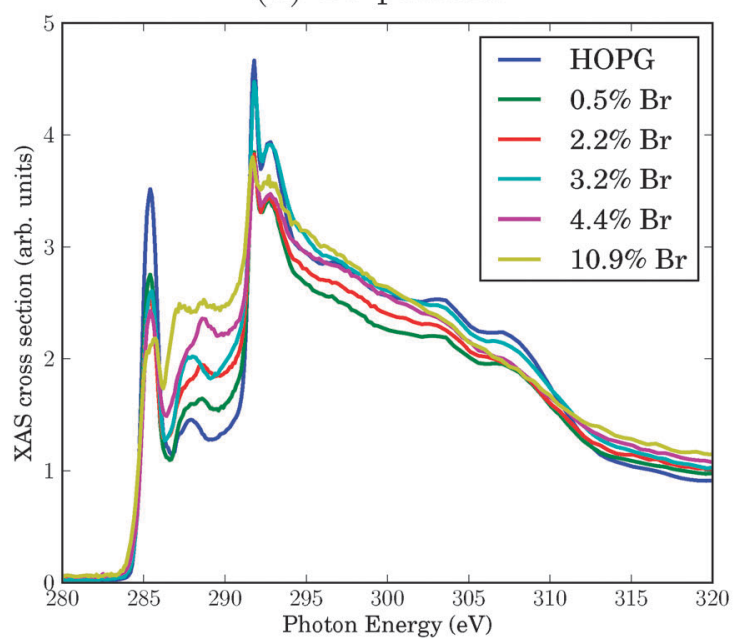

(b) Kr plasma

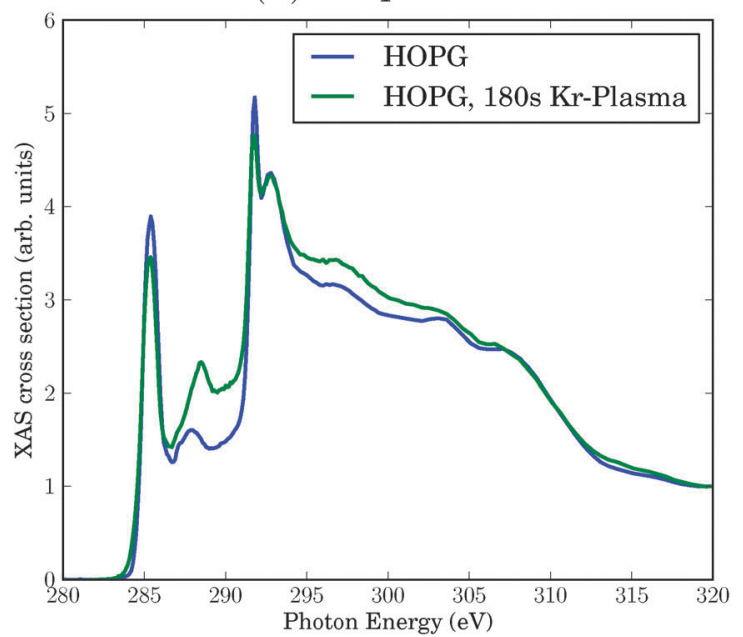

Fig. 1 Experimental C K-edge NEXAFS spectra of HOPG, treated with a bromine plasma leading to variable Br concentrations determined by XPS (a), or a krypton plasma applied for $180 \mathrm{~s}$ (b), respectively. ${ }^{6}$ The spectra for the pristine material are always shown for comparison. All spectra have been normalized as usual to the absorption jump such that the intensity at a photon energy of $330 \mathrm{eV}$ is $1 .^{7}$

effects (e.g., vacancy defects) or chemical effects (e.g., due to addition or substitution reactions involving bromine). To disentangle physical and chemical effects, in ref. 6 HOPG was also treated with a krypton plasma. $\mathrm{Kr}$ is chemically inert but may still cause physical damage. In Fig. 1(b), we compare the NEXAFS spectrum of pristine HOPG with a spectrum obtained after $180 \mathrm{~s}$ treatment with $\mathrm{Kr}$ plasma (see ref. 6 for details). Again, some characteristic changes are observed (we discuss changes only in the energy region up to about $294 \mathrm{eV}$ in what follows):

- The intensities of $\pi^{*}$ and $\sigma^{*}$ resonance intensities decrease slightly.

- The biggest change is found in the "fingerprint" region between about 286 and $290 \mathrm{eV}$, where Kr plasma treatment leads to higher intensities. (Also, a shift of the feature from $287.9 \mathrm{eV}$ (for HOPG), to $288.4 \mathrm{eV}$ (for Kr-HOPG) is observed.)

In passing we note that all experimental spectra have been arbitrarily normalized such that the intensity at a photon energy of $330 \mathrm{eV}$ is $1 .^{7}$

Also here, the precise atomic/molecular origin of spectral changes is largely unknown. In what follows we examine by means of electronic structure calculations, the two possible causes by which plasma treatment of graphite surfaces can influence their NEXAFS signatures: physical modification by creation of point and line defects, and, in case of Br plasma treatment, additional chemical modification by substitution and addition reactions of $\mathrm{Br}$ atoms. In both cases the hybridization of $\mathrm{C}$ atoms may change, from $\mathrm{sp}^{2}$ to $\mathrm{sp}^{3}$, and also the chemical environment will be affected, resulting in new spectral features.

In order to account for physical effects, we shall consider a single (graphene) layer, adopting models for various defect types whose character and energies have recently been investigated by density functional theory and by experiment in ref. 9 . We note that related work exists in the literature where NEXAFS spectra of graphene nano-sheets have been measured before and after treatment with an acid, which also introduces defects by bond cleavage in the $\mathrm{C}-\mathrm{C}$ network. ${ }^{10}$ In this work, electronic structure calculations (density-of-state curves) have been used to rationalize the experimental findings. Furthermore, Schiros et $a .^{11}$ measured and calculated the NEXAFS resonances of nitrogen-doped graphene. However, a detailed discussion of various types of defects and notably their impact on NEXAFS spectra, is still elusive. We shall further study models containing bromine, in order to make contact to the Br plasma experiments.

The paper is organized as follows. In the next Section 2 we shall describe details of the theoretical models, which are all based on density functional theory to calculate $\mathrm{C}$ K-edge NEXAFS spectra (and Br 3d XP spectra). In Section 3 and 4 results will be presented and discussed, first for NEXAFS of a single defect-free graphene layer as a reference, then for graphenes with "physical defects" and finally "chemical defects" involving bromine, respectively. A final Section 5 summarizes and concludes this work.

\section{Computational details}

In general, one can use two different approaches to characterize the electronic structure of graphene and defective variants of it. The first is based on a local cluster model, where molecular representatives are adopted to mimick a graphene layer (see, for example, ref. 12 and 13). Clearly, this introduces unwanted edge effects due to cutting of $\mathrm{C}-\mathrm{C}$ bonds and saturation with $\mathrm{H}$ atoms. The second approach uses periodic boundary conditions instead (see, for example ${ }^{14-16}$ ). In this way artificial boundaries are avoided, however, large unit cells may be necessary to model low-density defects and/or to avoid repeated interactions between defects. In what follows, we shall use periodic models for NEXAFS spectra, using large unit cells. For brominated species we will also compute $\mathrm{Br} 3 \mathrm{~d}$ ionization potentials to obtain peak positions of X-ray Photoelectron Spectra (XPS), via cluster models. 


\subsection{K-edge NEXAFS spectra}

For NEXAFS spectra, all calculations are based on periodic density functional theory (DFT) within the Kohn-Sham scheme, ${ }^{17}$ along with plane wave bases and pseudopotentials. Calculations were performed with the Quantum ESPRESSO program. ${ }^{18}$ The generalized gradient-corrected exchange-correlation functional $E_{\mathrm{xc}}$ due to Perdew, Burke, Ernzerhof (PBE) was used, ${ }^{19}$ and a plane-wave energy cutoff $V_{\mathrm{c}}=60$ Ry was adopted.

Two different types of atomic pseudopotentials were utilized (as described in http://www.quantum-espresso.org). For "normal" $\mathrm{C}$, as well as $\mathrm{H}$ and $\mathrm{Br}$ we adopted norm-conserving pseudopotentials of the Martins-Troullier type (keywords C.pbemt_gipaw.UPF, H.pbe-mt_fhi.UPF and Br.pbe-mt_fhi.UPF, respectively). ${ }^{20}$ To determine $\mathrm{C}$ K-edge spectra, we also adopt special pseudopotentials for the $\mathrm{C}$ atoms of interest, which contain a C 1s core hole (keyword C.star1s-pbe-mt_gipaw.UPF). This latter procedure corresponds roughly to replacing a neutral $\mathrm{C}$ pseudopotential by an $\mathrm{N}$-like pseudopotential, and considering five instead of four valence electrons for the target atom. As a consequence, the supercell remains uncharged. All calculations are done in spin-unpolarized fashion, even for odd numbers of electrons.

For our graphene models, we used $7 \times 7$ supercells in a slab geometry, with individual layers separated along the perpendicular direction by a large vacuum gap of $15 \AA$. A defect-free elementary cell contains $98 \mathrm{C}$ atoms. Four different types of "physical defects" were considered: (1) a Stone-Wales defect, (2) a single-vacancy defect, (3) a double-vacancy defect, and (4) a line-defect. Defects (1) and (3) can be realized by all-C models, while (2) and (4) contain also saturating $\mathrm{H}$ atoms. Further, two different "chemical defects" containing both $\mathrm{H}$ and up to two $\mathrm{Br}$ atoms in addition to $\mathrm{C}$, were considered. We optimized the geometry and the cell parameters for all models at the $\Gamma$ point (i.e., using a single $k$-point). Structures will be displayed below.

To obtain NEXAFS spectra, we adopted a pseudopotentialbased, iterative procedure as suggested elsewhere. ${ }^{21}$ Accordingly, the X-ray absorption cross section is calculated from the Golden Rule expression

$$
\sigma(\omega)=4 \pi \alpha \hbar \omega \sum_{\mathrm{f}}\left|M_{\mathrm{i} \rightarrow \mathrm{f}}\right|^{2} \delta\left(E_{\mathrm{f}}-E_{\mathrm{i}}-\hbar \omega\right)
$$

where $\alpha$ is the fine-structure constant, $\hbar \omega$ the excitation energy, and $E_{\mathrm{f}}$ and $E_{\mathrm{i}}$ are energies of final and initial states. Further, in the dipole approximation (which is valid for photon energies relevant here), the transition matrix element connecting initial state $\psi_{\mathrm{i}}$ with final state $\psi_{\mathrm{f}}$ is

$$
M_{\mathrm{i} \rightarrow \mathrm{f}}=\left\langle\psi_{\mathrm{f}}|\underline{\varepsilon} \cdot \underline{r}| \psi_{\mathrm{i}}\right\rangle
$$

where $\underline{\varepsilon}$ is the polarization vector of the photon beam.

In our case, $\psi_{\mathrm{i}}$ is a core state, i.e., a $\mathrm{C}$ 1s orbital which can be reconstructed from the ground state density and non-core excited pseudopotentials (see below). Further, $\psi_{\mathrm{f}}$ is a final state, i.e., an excited empty state obtained from solving the Kohn-Sham equations in which the pseudopotential for the one C-atom of interest has been replaced by the special pseudopotential with a $\mathrm{C} 1 \mathrm{~s}$ core hole. The explicit, direct calculation of all possible final states at every $k$-point can be costly. We therefore use a two-step procedure. First, the charge density, with the core-hole pseudopotential for one C-atom is obtained by directly solving the Kohn-Sham equations selfconsistently, on a $4 \times 4$ Monkhorst $k$-point grid. ${ }^{22}$ In a second step, an iterative procedure based on a Lanczos recursion method as suggested in ref. 21 is used, to determine empty final states iteratively, adopting a denser Monkhorst $k$-point grid, $10 \times 10$.

This method is implemented in the XSpectra program $^{23}$ as used here, and which also gives the cross section $\sigma$ as a function of photon energy. The program computes transition amplitudes $M_{\mathrm{i} \rightarrow \mathrm{f}}$ from all-electron functions $\psi_{\mathrm{i}}$ and $\psi_{\mathrm{f}}$. How the latter can be reconstructed when PAW-type (Projected Augmented Wave) pseudopotentials $^{24}$ are used, is described in detail in ref. 21. Also a broadening factor $\gamma$ has to be specified to represent the delta functions in eqn (1), which we choose as $\gamma=0.2 \mathrm{eV}$ throughout. Finally, since the final and initial state energies have been calculated from (different) pseudopotentials, the computed spectra were shifted such that the theoretical C $1 \mathrm{~s} \rightarrow \pi^{*}$ resonance for pristine graphene coincide with the experimental value, of $285.4 \mathrm{eV}$. Note that in our approach many-body corrections to Kohn-Sham energies ${ }^{25}$ or electron-phonon couplings are absent, however, for a comparative study of similar systems we expect this method to be sufficiently accurate.

\subsection{Br 3d XP spectra}

In NEXAFS, the final states are bound in contrast to XPS where they are part of the ionization continuum. The XPS measurements of ref. 6 on Br plasma treated species are not only useful to monitor the $\mathrm{Br}$ content of samples, but also to unravel structural details. To make contact to experiment, we have also simulated XP (Br 3d) spectra for brominated graphene models, using the $\Delta$-Kohn-Sham $\left(\Delta\right.$-KS) method. ${ }^{26}$ Cluster (rather than periodic) models for XP spectra are adopted in this case, to determine core ionization potentials

$$
\mathrm{IP}_{\mathrm{i}}=E_{\text {ion }}(\mathrm{i})-E_{\text {neu }} .
$$

Here, $E_{\text {ion }}(\mathrm{i})$ is the energy of a cation obtained after removing an electron from a $3 \mathrm{~d}$ orbital of a $\mathrm{Br}$ atom ( $\mathrm{i}$ is a combined orbital and atom index). $E_{\text {neu }}$ is the energy of the neutral cluster. In order to compute these quantities, we applied the $\Delta$-KS methodology as implemented in the StoBe program. ${ }^{27}$ In a preparation step, a neutral cluster model comprising $\mathrm{C}, \mathrm{Br}$, and $\mathrm{H}$ atoms was geometry-optimized with Gaussian09, ${ }^{28}$ using the PBE exchange-correlation functional, the D3 dispersion ${ }^{29}$ energy correction, and a $6-311 \mathrm{G}^{* *}$ atomic orbital basis set. ${ }^{30}$ Using this geometry, two separate KS calculations were done with StoBe. First, the energy $E_{\text {neu }}$ of the neutral cluster was recalculated with the PBE xc-functional, using now an effective core potential for $\mathrm{C}$ atoms together with the corresponding triple- $\zeta$ basis set as implemented in StoBe, an effective core potential for $\mathrm{Br}$ atoms (with 18 core electrons), and its corresponding double- $\zeta$ basis set. For $\mathrm{H}$, a double- $\zeta$ basis set was used. In a second step, the cation and $E_{\text {ion }}(\mathrm{i})$ was self-consistently determined by an 
unrestricted KS (UKS) calculation, by adopting the so-called "supersymmetry" option to remove an electron from a selected $3 \mathrm{~d}$-orbital of a selected $\mathrm{Br}$ atom and preserving this occupation pattern during the entire SCF procedure. The obtained ionization potentials are interpreted as peak positions of XP spectra of brominated graphene. Note that our approach yields no XPS intensities. Also, spin-orbit splitting of $\mathrm{Br} 3 \mathrm{~d}$ orbitals is neglected.

\section{Results and discussion}

\subsection{Single, defect-free graphene sheet}

First, we present the theoretical NEXAFS spectrum of a single sheet of graphene and compare it with experiment ${ }^{6}$ in Fig. 2(a). To make this comparison more meaningful, the theoretical and experimental spectra were normalized such that the maximum intensity of the resonance feature at $285.4 \mathrm{eV}$ is 1 .

The resonance at $285.4 \mathrm{eV}$ represents the $\mathrm{C} 1 \mathrm{~s} \rightarrow \pi^{*}$ excitation. Experiment and theory agree here well by construction with respect to intensity and position. Also the width fits very well. The nature of this resonance as being due to C $1 \mathrm{~s} \rightarrow \pi^{*}$ transitions is proven by the fact that the in-plane contribution $(x, y)$ to the cross section is practically zero, while the out-of-plane contribution $(z)$ makes the entire spectrum. This can be seen from "polarized" results in Fig. 2(b).

The second interesting photon energy interval is between $286 \mathrm{eV}$ and $289 \mathrm{eV}$, the energy range which is known to be sensitive to chemical modifications of graphene or graphite surfaces $^{10,31}$ (the "fingerprint" region of above). Both in experiment and theory spectral features with weak intensity appear there, more clearly so in experiment than in theory. These features are also mostly due to $\mathrm{C} 1 \mathrm{~s} \rightarrow \pi^{*}$ excitations, as evident from the polarized spectra in Fig. 2(b).

The third photon energy interval we wish to discuss is around 292-293 eV, which corresponds to the lowest-energy C 1s $\rightarrow \sigma^{*}$ excitation. The $\sigma$-character can be seen from the $(x, y)$ (in plane) polarization of the signal, $c f$. Fig. 2(b). Note that location and overall width of the theoretical, $\sigma^{*}$ resonance agrees with the experimental one, however, the double-resonance structure is hardly apparent in theory. Most strikingly, the intensity of this resonance is too high. It must be noted, however, that the intensity is a function of the broadening parameter adopted in the calculation. We will also see that the intensity of this resonance depends sensitively on the presence of defects. By using fixed broadening and "absolute" signals from now on, we hope to elucidate meaningful trends emerging from various models of defective graphene.

Further excitations arise in the energy range between $295 \mathrm{eV}$ and $330 \mathrm{eV}$, again both in theory and experiment. The polarized calculations show that these are both of $\sigma$ - (in-plane) and $\pi$ - (out-of-plane) character. According to Fig. 2(b), the $\sigma$-character dominates up to about $312 \mathrm{eV}$, and the $\pi$-character at photon energies above.

With the present choice of the broadening $\gamma$, the theoretical spectrum appears to be more structured than experiment, cf. Fig. 2(a). Of course, using a larger (or energy-dependent) broadening factor would improve agreement between theory and experiment, but we refrain here from adapting $\gamma$.

In summary, the theoretical spectrum is in reasonably good agreement with experiment, with the exception of a too intense $\sigma^{*}$ resonance at around $292 \mathrm{eV}$.

\section{2 "Physical" defects}

We now study the effect of "physical" structural defects in graphene on C K-edge NEXAFS spectra. We shall consider the four defect types as described later in this section, which are selected based on energy criteria as outlined in earlier work. ${ }^{9}$ In our case those physical defects originate from the bombardment of graphene surface by plasma particles. The energy of these "projectiles" is sufficiently high not only to break bonds but also to sputter atoms out of the graphene lattice. (a)

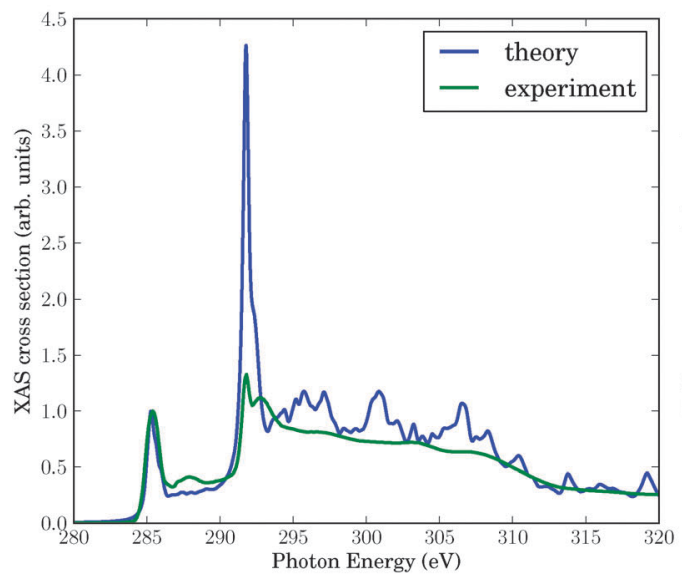

(b)

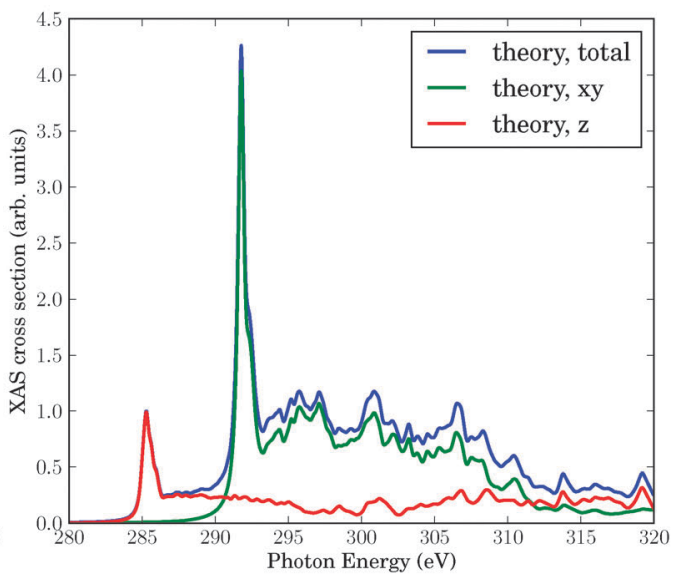

Fig. 2 (a) Calculated NEXAFS spectrum of graphene compared to experiment. The theoretical curve was obtained with a constant broadening factor $\gamma=$ $0.2 \mathrm{eV}$. Both spectra are normalized such that the intensity is 1 for the $\pi^{\star}$ resonance at $285.4 \mathrm{eV}$. (b) Theoretical spectrum: in-plane ("xy") and out-of plane (" $z$ ") contributions to the total NEXAFS spectrum. 

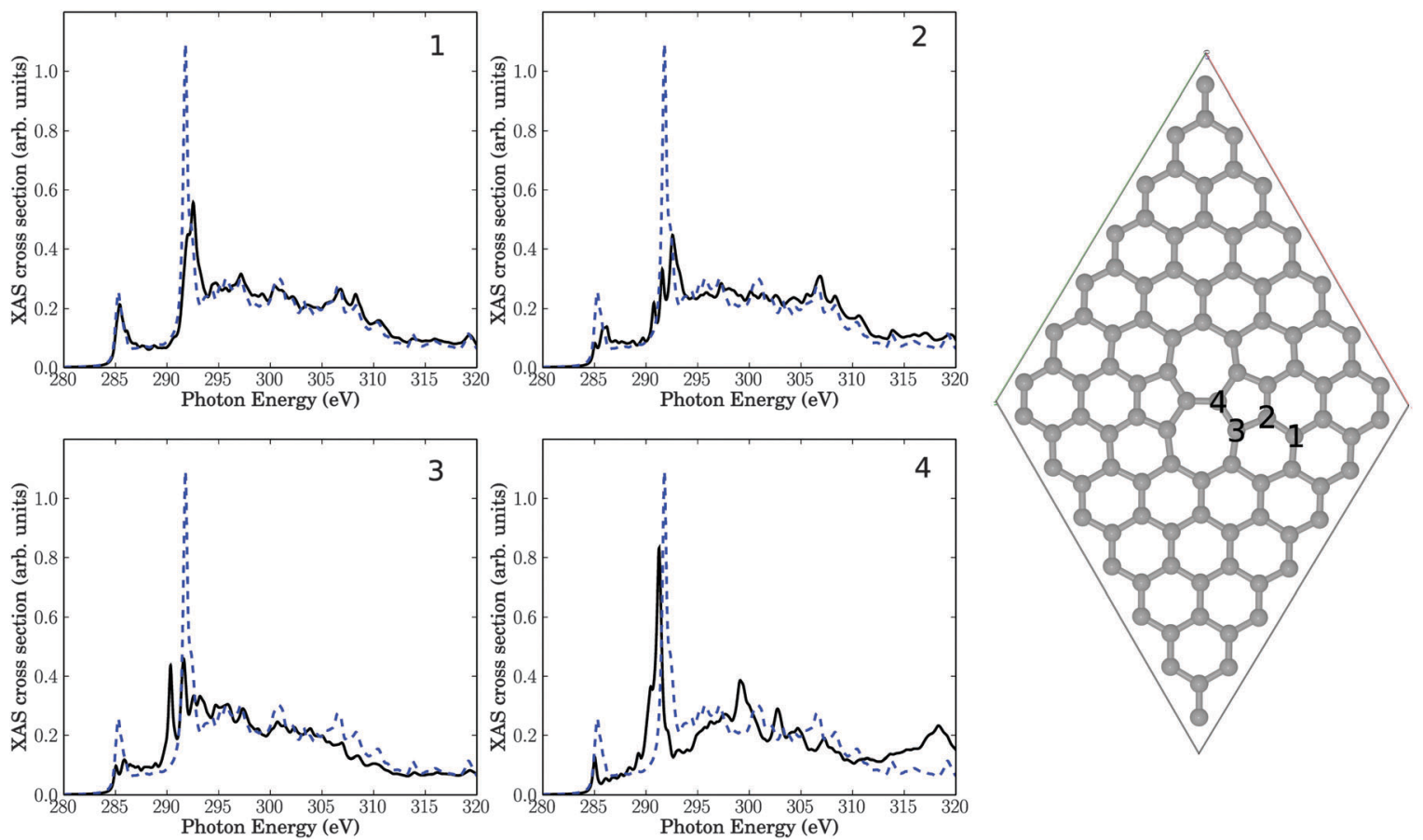

Fig. 3 Right: Stone-Wales (55-77) defect in a $7 \times 7$ cell. The four panels show NEXAFS spectra corresponding to core-hole creation in atoms $1-4$, located close to the defect (see atom numbering). Full, black lines: computed NEXAFS spectra, dashed, blue lines: the theoretical spectrum of unperturbed graphene for comparison.

As mentioned above, all structures below are fully geometryoptimized on the PBE Kohn-Sham level of theory.

3.2.1 Stone-Wales defect. As a first structural defect we refer to a so-called Stone-Wales defect (called SW(55-77) in ref. 9). This defect is created by rotating two carbons by 90 degrees, with a formation energy of $\sim 5 \mathrm{eV} .^{9}$ The manifestation of this rotation is the appearance of two five-rings and two seven-rings. For the SW defect, no dangling bonds are created and all $\mathrm{C}$ atoms remain $\mathrm{sp}^{2}$-hybridized. We consider a single such defect in our $7 \times 7$ unit cell, as shown in Fig. 3.

Also depicted in the figure are four NEXAFS spectra, corresponding to excitation out of the $\mathrm{C} 1$ s orbital of either $\mathrm{C}$ atom 1 , 2,3 , or 4 . These $\mathrm{C}$ atoms are located in the center of three 6-rings (atom 1), two six-rings and one five-ring (atom 2), one six-ring, one five-ring and one seven-ring (atom 3), and one fivering and two seven-rings (atom 4), respectively. Atom number 4 is in the center of the defect, while atoms 3,2 and 1 are increasingly remote from the defect center. For comparison, in every spectrum the theoretical NEXAFS spectrum for defectfree graphene is shown. In contrast to Fig. 2, we give absolute, non-rescaled XAS cross sections from now on. In an actual experiment, the resulting spectrum would be the average over all $\mathrm{C}$ atoms in the cell.

The first observation is that all spectra look different. This is evidence for the sensitivity of NEXAFS to the chemical environment of an atom. We notice that the character of the spectra changes the closer the atom is to the defect center. Nevertheless, even in the case of atom 1 (with the same local environment as in defect-free graphene), the NEXAFS spectrum looks different from pure graphene, indicating effects beyond nearest neighbours.
The $\pi^{*}$ resonance, located for unperturbed graphene at $285.4 \mathrm{eV}$ is observable in all four spectra. However, the intensity of this resonance is reduced, except for atom 1 which is farthest away from the defect. The intensity decreases the nearer the atom to the center of the defect is. We also observe a splitting of that resonance for atoms 2 and 3.

New resonances arise in the region between $286.8 \mathrm{eV}$ and $290.8 \mathrm{eV}$ in some cases. Recall that this "fingerprint" region is the range where changes of NEXAFS spectra were found after krypton treatment. Especially atom numbers 3 and 4, which are close to the defect, show new absorption features near $290.3 \mathrm{eV}$. Closer analysis shows that the two resonances slightly above $290 \mathrm{eV}$ for atom 3, for example, are both of $\sigma$ symmetry, i.e., the original $\sigma^{*}$ signal is shifted to lower photon energies and splits. The splitting may be explained by the fact that $\mathrm{C}$ atom 3 has now (three) slightly different $\mathrm{C}-\mathrm{C}$ bondlengths to neighbour atoms, hence, at least in a localized picture different $\sigma^{*}$ orbital energies emerge. The shift to lower photon energies could be a result of the fact that most of the bonds around $\mathrm{C} 3$ are elongated w.r.t. defect-free HOPG (1.42 $\AA$ ), leading to a smaller $\sigma-\sigma^{*}$ splitting and hence a lower final-state energy. It should be noted that this interpretation is not fully unambiguous (one out of three $\mathrm{C}-\mathrm{C}$ bonds of $\mathrm{C}$ atom 3 is shortened relative to the $\mathrm{C}-\mathrm{C}$ bond length of HOPG), and, also, the overall changes in the "fingerprint" region are relatively modest.

An interesting observation is that in three of four spectra the high-intensity $\sigma^{*}$ resonance at around $292 \mathrm{eV}$ looses intensity to a significant extent. As a consequence, the intensity ratios between the $\sigma^{*}$ resonance and other resonances decrease, in some cases (for atoms 2 and 3) quite dramatically. Since the 
relative $\sigma^{*}$ resonance height in unperturbed graphene was too large compared to experiment ( $c f$. Fig. 2), such disagreement may therefore be due to defects which are unavoidable in a real crystal, at finite temperature. Closer inspection reveals that the $\sigma^{*}$ signal of carbon 2 shows two additional resonances below the main signal. Atom 3 exhibits one additional resonance. The $\sigma^{*}$ signal of atom 4 shifts to slightly lower photon energies and develops a shoulder.

In general, the shift/splitting of the $\sigma^{*}$ signal is sensitive to $\mathrm{C}-\mathrm{C}$ bond lengths and can in fact be used as a tool to measure them. ${ }^{32}$

Relatively large changes of the spectra of defective structures are observed in the high-energy regions around $320 \mathrm{eV}$, at least for atom 4

In summary, a SW defect has some effect on theoretical NEXAFS spectra of graphene, with features consistent with experimental signatures after plasma treatment of HOPG which inherently leads to such defects.
3.2.2 Single-vacancy defect. The second perturbation we discuss is a single-vacancy defect. This defect, called $V_{1}(5-9)$ in ref. 9 , is created by removing a single carbon atom. As shown in Fig. 4, this leads to the formation of a five- and a nine-membered ring. One atom (atom 9 in Fig. 4) has a dangling bond, which we saturate here by two $\mathrm{H}$ atoms. Atom no. 9 thus becomes $\mathrm{sp}^{3}$-hybridized, while all other $\mathrm{C}$ atoms remain $\mathrm{sp}^{2}$-hybridized. The saturation of dangling bonds with hydrogen can hardly be avoided in practice. The formation energy of an undecorated $\mathrm{V}_{1}$ defect is about $7.5 \mathrm{eV}$ according to DFT calculations. ${ }^{9}$

The resulting structure and NEXAFS spectra of atoms near the defect are presented in Fig. 4. Again we see that all spectra are different from the unperturbed graphene layer. Specifically, the following observations are made.

- Atom number 9, the $\mathrm{sp}^{3}$-hybridized $\mathrm{C}$ atom, looses intensity of the $\pi^{*}$ resonance at $285.4 \mathrm{eV}$, which is not unexpected. Closer inspection shows, however, that the $z$-component of the intensity is not fully lost but partially shifted (to about $288 \mathrm{eV}$ ),
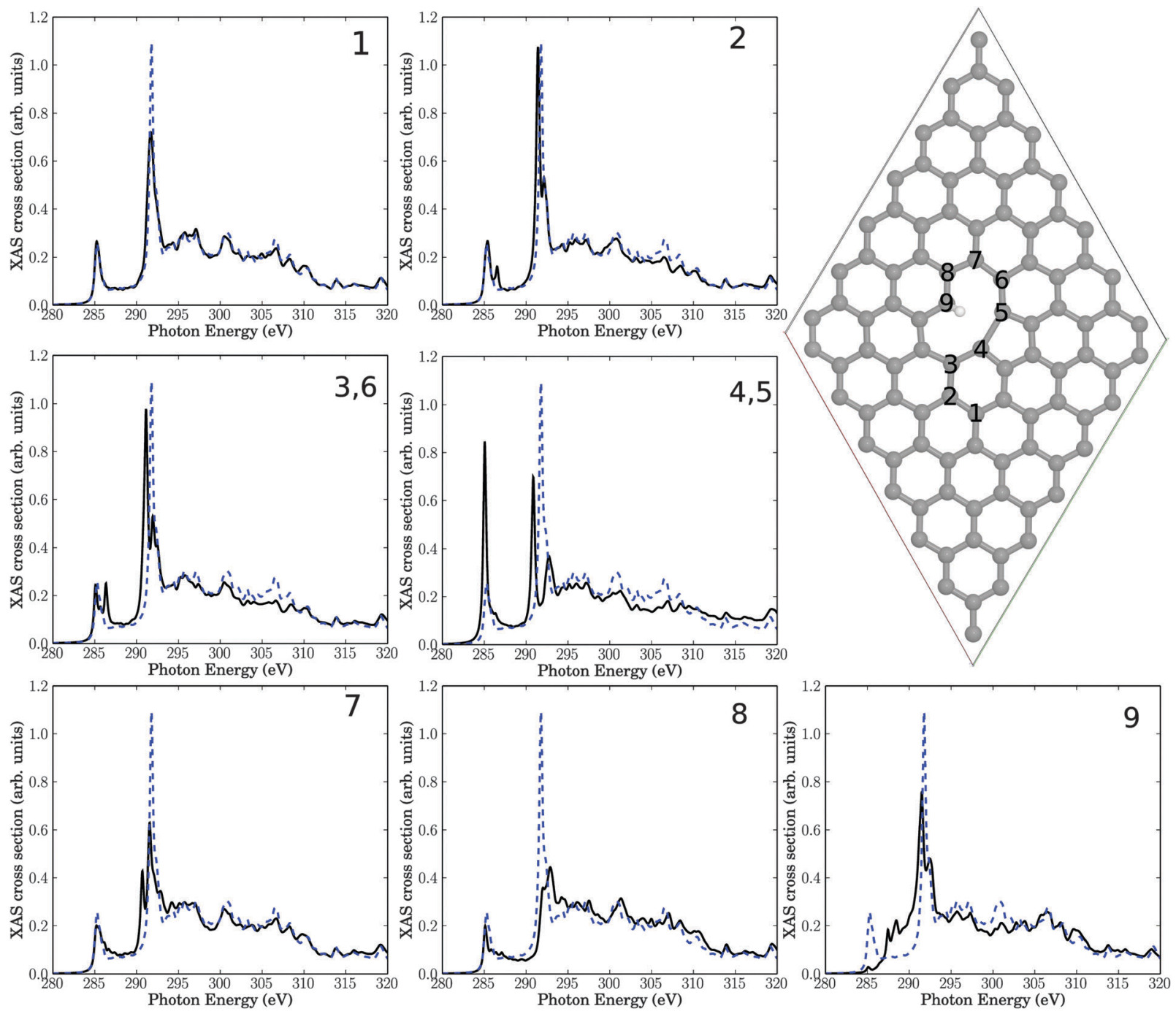

Fig. 4 Upper right: single-vacancy $\left(V_{1}(5,9)\right)$ defect, decorated with two $\mathrm{H}$ atoms (both at $C$ atom 9), in a $7 \times 7$ cell. The seven insets show NEXAFS spectra corresponding to core-hole creation in atoms 1-9, located close to the defect. Full, black lines: computed NEXAFS spectra, dashed, blue lines: the theoretical spectrum of unperturbed graphene for comparison. 
i.e., into the "fingerprint" region (see below). This intensity should not be interpreted as being $\pi$-like, though, because C9 has a fully saturated albeit three-dimensional, atomic neighbourhood.

- Also the neighbour atom 8 shows a reduced $\pi^{*}$ intensity. In some cases, we observe a splitting of the $\pi^{*}$ signal at $285.4 \mathrm{eV}$ (for example, for atoms 2, 3(6)). Other spectra's $\pi^{*}$ resonances remain largely unaffected (for example, atoms 1,7 ), or the $\pi^{*}$ intensity increases (for example for atom 4 (equivalent to 5)).

- For the $\mathrm{sp}^{3}$ atom 9 intensity appears in the "fingerprint" region between $286.8 \mathrm{eV}$ and $290.8 \mathrm{eV}$. Also atom 4 (5) and to a lesser extent $3(6)$ and 7 show an intensity gain in this region, close to the $\sigma^{*}$ resonance.

- The original $\sigma^{*}$ resonance appears to be reduced in intensity in most cases (atoms 1, 4 (5), 7, 8, 9), sometimes splitted (e.g., atoms 2, 3 (6), 4 (5), 7, 9).

- Atoms 2 and 3, which are farthest away from the defect, are only slightly affected.
- In all spectra, the high-energy region (above about $310 \mathrm{eV}$ ) is almost unchanged.

Overall, these findings are similar to the Stone-Wales defect, with the dominant effects: reduction of the $\sigma^{*}$ resonance and new resonant features in the "fingerprint" region between $\pi^{*}$ and $\sigma^{*}$. In addition to the observations for SW the formation of a sp ${ }^{3}$-hybridized $\mathrm{C}$ atom leads to a loss of $\pi^{*}$ resonant features, in particular at that atom. Once again, these findings are consistent with experimental features following plasma treatment.

A few additional tests have been carried out for the singlevacancy case. First, it has been stressed that defects in graphene can lead to long-ranged strain fields. ${ }^{33}$ In order to study a possible effect of long-range order on NEXAFS, we have also used for the single-vacancy a larger supercell than $7 \times 7$ cell, namely a $9 \times 9$ cell with a $\mathrm{V}_{1}(5,9)$ defect. However, no clear differences w.r.t. to Fig. 4 (black curves) could be found (see Fig. S1 in the ESI $\dagger$ ), which is why the $7 \times 7$ cell was used throughout. We also
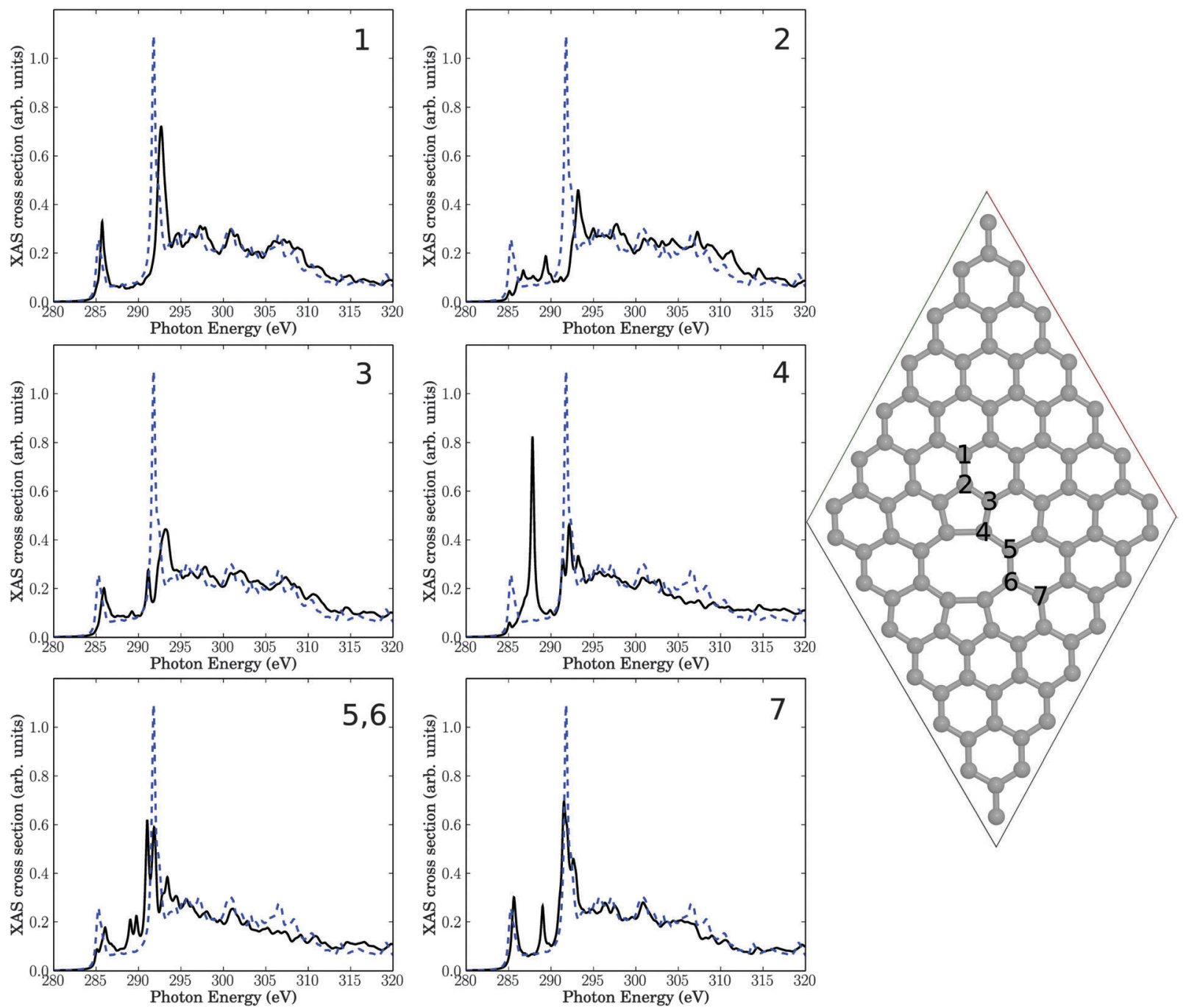

Fig. 5 Right: double-vacancy $\left(V_{2}(5-8-5)\right)$ defect in a $7 \times 7$ cell. The six insets show NEXAFS spectra corresponding to core-hole creation in atoms $1-7$, located close to the defect. Full, black lines: computed NEXAFS spectra, dashed, blue lines: the theoretical spectrum of unperturbed graphene for comparison. 
calculated, for the $7 \times 7$ cell the spectra for a saturation with only one saturating hydrogen atom (leaving atom no. $9 \mathrm{sp}^{2}$ hybridized). This leads to a decrease of the resonance intensity in the fingerprint region for carbon atom no. 9 and minor changes for the other carbons, as reported in the ESI $\dagger$ (Fig. S2).

3.2.3 Double-vacancy defect. This defect is created by removing two neighboring carbon atoms. This leads to an eight-ring connecting two five-rings, which is why this point defect was called $\mathrm{V}_{2}(5-8-5)$ in ref. 9. Other double-vacancy defects have been considered in that reference. According to ref. 9, the formation energy of $\mathrm{V}_{2}(5-8-5)$ is about $8 \mathrm{eV}$. In the defect, no dangling bonds appear which could be saturated, and all $\mathrm{C}$ atoms remain $\mathrm{sp}^{2}$-hybridized. The structure of the defect and the spectra of atoms near it are displayed in Fig. 5.

This structure serves as another support of our hypothesis, that defects may be responsible for the observed intensity changes upon plasma treatment. Specifically, out of the seven investigated carbon atoms near the defect, four show additional resonances in the "fingerprint" region. Especially atom number 4, which is in the center of the defect, exhibiting a sharp resonance with high intensity. The signals in the "fingerprint" region for atoms 4, 5, and 6 , are all of $\sigma$ symmetry. As a consequence, the resonance intensity of $\sigma^{*}$ at around $292 \mathrm{eV}$ is reduced for all investigated atoms compared to unperturbed graphene, proving once more the sensitivity of this resonance w.r.t. defects. Again, effects on the NEXAFS spectra of atoms farther away from the defect center, e.g., atom 1 , are small.

3.2.4 Line defect. When we remove four neighbouring $\mathrm{C}$ atoms along a line, we obtain a line defect. Saturating the dangling bonds with (eight) hydrogens a decorated line-defect as shown in Fig. 6 emerges. All $\mathrm{C}$ atoms remain $\mathrm{sp}^{2}$-hybridized. This structure is not only a model for a line defect, it can also be viewed as a model for decorated edges of graphene flakes. In Fig. 6, we also show NEXAFS spectra for 8 selected atoms.

Again, the general observations are similar to those of above: most atoms close to the defect are characterized by reduced
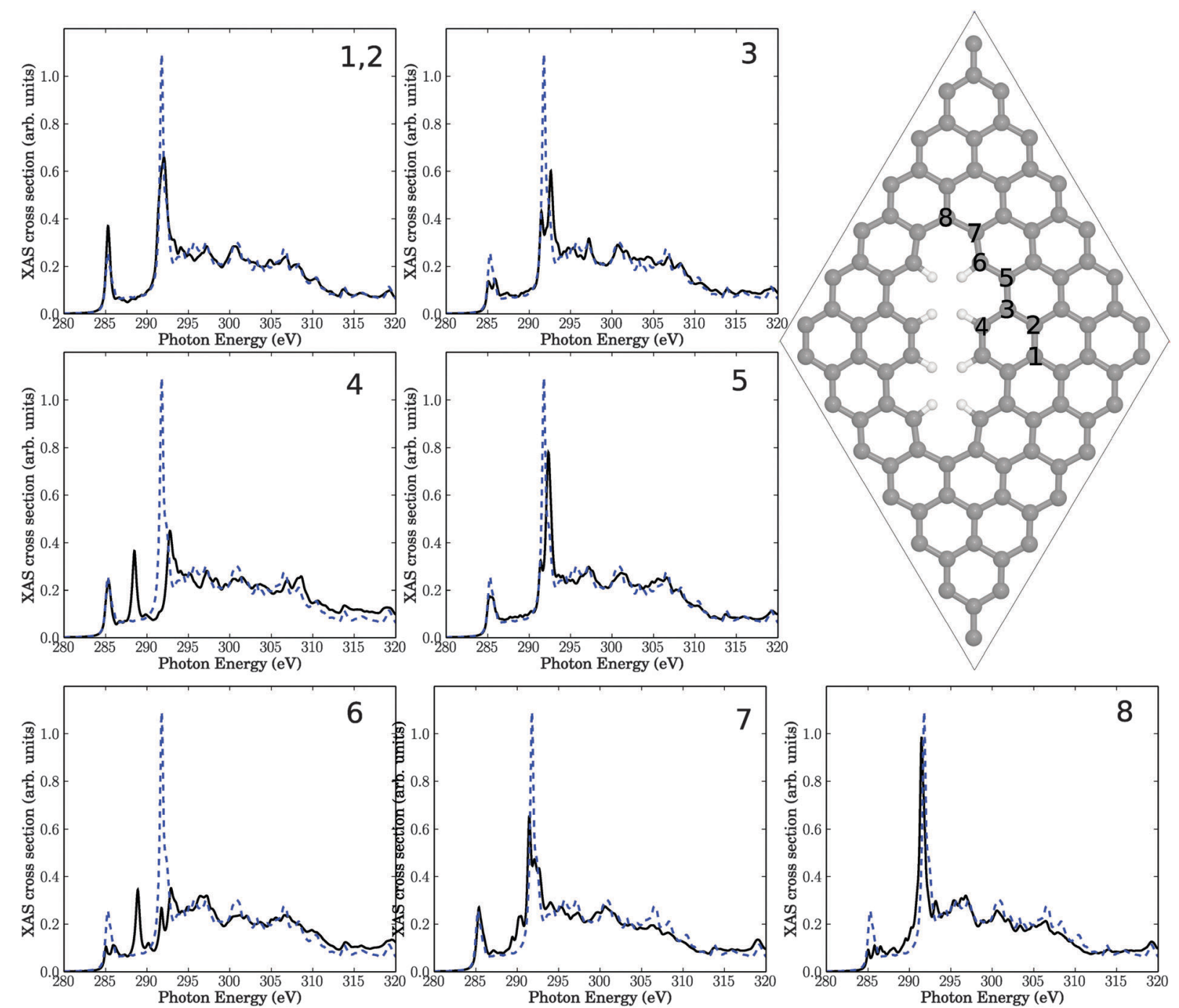

Fig. 6 Upper right: decorated line defect in a $7 \times 7$ cell. The seven insets show NEXAFS spectra corresponding to core-hole creation in atoms $1-8$, located close to the defect. Full, black lines: computed NEXAFS spectra, dashed, blue lines: the theoretical spectrum of unperturbed graphene for comparison. 
$\pi^{*}$ and $\sigma^{*}$ intensities, and for some atoms (in particular atoms 4 and 6) clear additional resonances appear in the "fingerprint" region. The latter two atoms are the ones which carry $\mathrm{H}$ atoms. Closer analysis for atom 4 for example shows again, that the "additional" resonances are actually shifted and split, $\sigma^{*}$ resonances. The different resonances arise from different $\mathrm{C}-\mathrm{C}$ bond lengths (compared to defect-free HOPG), and the new resonances can now be lower or slightly higher in energy than the defect-free $\sigma^{*}$ signal.

\section{4 "Chemical" defects}

\subsection{XP Br 3d spectra}

In this section we consider graphene layers containing bromine, mimicking the situation encountered after $\mathrm{Br}_{2}$ plasma treatment. There is a large variety of how $\mathrm{Br}_{2}$ can react with graphene. Among various principal possibilities ${ }^{6}$ are the formation of covalent bonds between $\mathrm{Br}$ and $\mathrm{C}$ by nucleophilic substitution at plasma induced defects with $\mathrm{sp}^{3}$-hybridized $\mathrm{C}$ atoms. The same can be achieved by electrophilic or radical addition on $\mathrm{sp}^{2}$-hybridized $\mathrm{C}$ atoms $/ \mathrm{C}=\mathrm{C}$ double bonds. Finally, non-covalent interactions between $\mathrm{Br}$ or $\mathrm{Br}_{2}$ with the $\pi$-electron system of graphene are possible, as well as intercalations of bromine between graphene layers.

The experimental $\mathrm{Br} 3 \mathrm{~d}$ XP spectra of $\mathrm{Br}_{2}$-plasma treated graphene show at low $\mathrm{Br}$ concentrations, at least two co-existing $\mathrm{Br}$ species at $3 \mathrm{~d}_{5 / 2}$ binding energies of around $70.5 \mathrm{eV}$ and $68.4 \mathrm{eV}$, respectively. ${ }^{6}$ The latter, low-binding energy species looses intensity with increasing Br load. A preliminary assignment of the $70.5 \mathrm{eV}$ peak was to be due to covalent $\mathrm{C}-\mathrm{Br}$ bond formation where $\mathrm{C}$ is $\mathrm{sp}^{3}$-hybridized. The low-energy peak at $68.4 \mathrm{eV}$ was tentatively interpreted as being due to $\mathrm{Br}$ binding to $\mathrm{sp}^{2}$-hybridized $\mathrm{C}$ atoms. ${ }^{6}$

To test this hypothesis, XP spectra were calculated for four different cluster models as shown in Fig. 7. All models are derived from a $\mathrm{C}_{96}$ motif saturated at the edges with $26 \mathrm{H}$ atoms, by adding $\mathrm{Br}$ atoms, substituting $\mathrm{H}$ with $\mathrm{Br}$ and/or creating line defects. As before, also for the plasma experiments of ref. 6 hydrogenation of defects is practically unavoidable. In particular, Fig. 7(a) shows a scenario where two $\mathrm{Br}$ atoms reacted with an intact surface forming $\mathrm{C}-\mathrm{Br}$ bonds to (now) $\mathrm{sp}^{3}$-hybridized $\mathrm{C}$ atoms. Fig. 7(b) shows a similar case with two neighbouring $\mathrm{Br}$ atoms forming covalent bonds to $\mathrm{sp}^{3}$-hybridized $\mathrm{C}$ atoms, however, now with at least one of them being close to a (partially $\mathrm{H}$-decorated) defect. Fig. 7(c) stands for a single $\mathrm{Br}$ atom with a covalent bond to a $\mathrm{sp}^{2}$-hybridized $\mathrm{C}$ atom as part of a defect. Finally, Fig. 7(d) shows a single Br atom forming a covalent bond to a $\mathrm{sp}^{2}$-hybridized $\mathrm{C}$ atom at a $\mathrm{H}$-saturated edge of the cluster. Using the methodology of above, we calculated ionization energies for $\mathrm{Br} 3 \mathrm{~d}$ for all species.

From Table 1 we note that indeed, (averaged) 3d ionization potentials are substantially higher for $\mathrm{Br}$ atoms bonded to $\mathrm{sp}^{2}$-hybridized carbons at defects (c) or edges (d), compared to $\mathrm{Br}$ atoms attached to $\mathrm{sp}^{3}$-hybridized $\mathrm{C}$ atoms $(\mathrm{a}, \mathrm{b})$. The latter show a lower $3 \mathrm{~d}$ binding energy, in particular those attached to a formerly defect-free surface (a). The range of averaged $\mathrm{Br}$ $3 \mathrm{~d}$ ionization potentials is between $2-3 \mathrm{eV}$, not unlike the experimental range. ${ }^{6}$ (In passing we note that absolute XPS values in experiment are red-shifted with respect to our $\Delta$-KS values by about $4-6 \mathrm{eV}$.) Note also that there is a distribution of $3 \mathrm{~d}$ binding energies even for single $\mathrm{Br}$ atoms, due to non-degenerate (a)

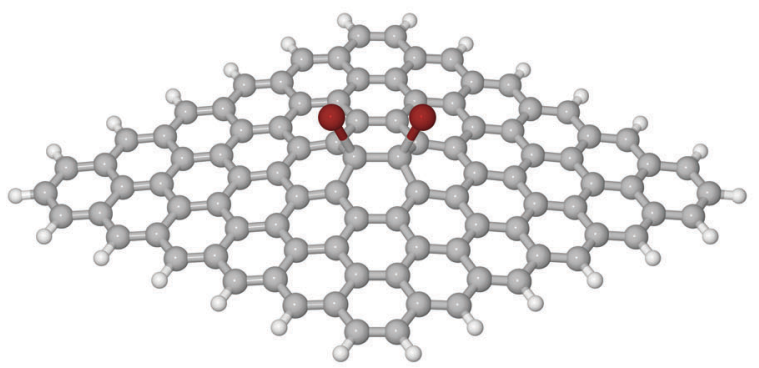

(c)

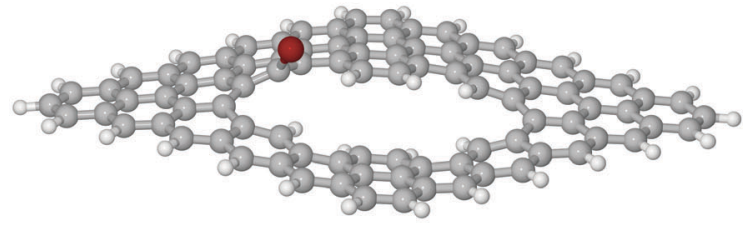

(b)

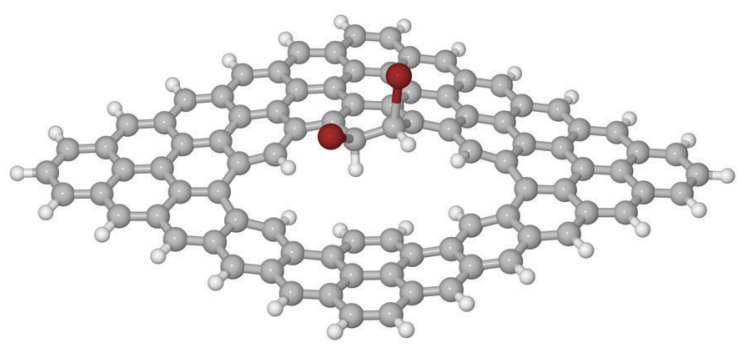

(d)

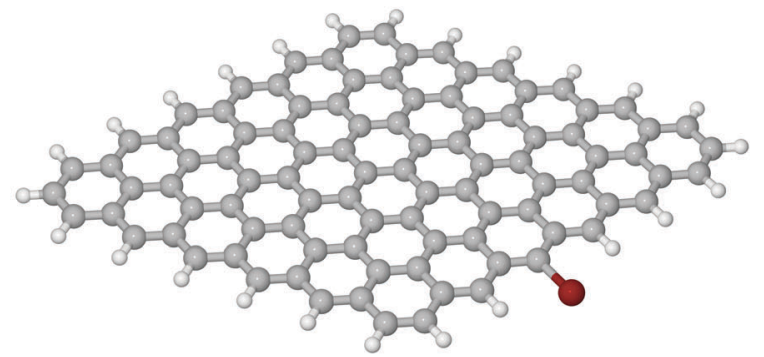

Fig. 7 Cluster models used for calculation of XP spectra. Br in red. See text for details. 
Table 1 XPS simulation for four different cluster models (cf. Fig. 7(a)-(d)), with the $\mathrm{C}-\mathrm{Br}$ binding character indicated. IP $(\mathrm{Br} 3 \mathrm{~d})$ is the averaged $3 \mathrm{~d}$ ionization potential of bromine, $\Delta \mathrm{IP}(\mathrm{Br} 3 \mathrm{~d})$ is the spread of $3 \mathrm{~d}$ ionization energies for a given cluster, and ESP $(\mathrm{Br})$ are average ElectroStatic Potential $^{34}$ atom charges for $\mathrm{Br}$

\begin{tabular}{lllll}
\hline Model & $\begin{array}{l}\text { (a) } \mathrm{sp}^{3}, \\
\text { "on-plane" }\end{array}$ & $\begin{array}{l}\text { (b) } \mathrm{sp}^{3}, \\
\text { at defect }\end{array}$ & $\begin{array}{l}\text { (c) } \mathrm{sp}^{2}, \\
\text { at defect }\end{array}$ & $\begin{array}{l}\text { (d) } \mathrm{sp}^{2}, \\
\text { at edge }\end{array}$ \\
\hline $\mathrm{IP}(\mathrm{Br} 3 \mathrm{~d}) / \mathrm{eV}$ & 74.11 & 75.04 & 75.99 & 76.45 \\
$\Delta \mathrm{IP}(\mathrm{Br} \mathrm{3d}) / \mathrm{eV}$ & 0.45 & 1.48 & 0.82 & 0.50 \\
$\operatorname{ESP}(\mathrm{Br})$ & -0.1695 & -0.0852 & -0.0042 & 0.0043
\end{tabular}

3d orbitals. This is indicated in Table 1 as a spread $\Delta$ for $3 \mathrm{~d}$ signals. We further see that the core binding energies correlate with effective atom charges of $\mathrm{Br}$ (in the form of ElectroStatic Potential (ESP) charges, calculated with the Merz-Sing-Kollman scheme $^{34}$ ): roughly, the more negative $\mathrm{Br}$, the lower the $3 \mathrm{~d}$ core ionization energy as expected.

The XPS experiments of ref. 6 can thus be interpreted as follows. At low $\mathrm{Br}$ doses, $\mathrm{Br}_{2}$ reacts with largely intact graphene layers, forming for example by electrophilic addition to $\mathrm{C}=\mathrm{C}$ bonds, structures like those in Fig. 7(a). The latter have a small 3d core ionization energy. With continued Br plasma treatment, the probability to create physical defects, e.g., point or line defects or edges increases, which then can react with hydrogen and/or bromine. Therefore, species like those in Fig. 7(b)-(d) will appear. Since these have larger $3 \mathrm{~d}$ core ionization energies, continued bromination leads to shift of the XP spectrum to higher 3d binding energies in agreement with experiment. ${ }^{6}$

It should also be stressed that structure 7(a) is much less stable than structures (b)-(c), which are related to physical defects: the cluster in Fig. 7(a) is in fact unstable at the PBE+D3/6-311G** level of theory, in contrast to (b)-(d). To arrive at the geometry of Fig. 7(a), the PBE0 hybrid functional ${ }^{35}$ with an admixture of exact exchange had to be used. Even then, the Br adsorption energy is small. Further, also with periodic KS calculations adopting the PBE functional, an arrangement analogous to Fig. 7(a) was unstable, $c f$. Section 4.2.

\subsection{NEXAFS spectra}

We then calculated C K-edge NEXAFS spectra for brominated graphene layers, using periodic DFT as outlined earlier. In particular the two structures shown in Fig. 8 and 9 were considered, both related to reaction of bromine at a decorated line defect, $c f$. Fig. 6 . In fact, as mentioned above, an analogue to Fig. 7(a) was not found to be stable using periodic DFT on the PBE level of theory, neither for 1,2 nor for 1,4 addition of $\mathrm{Br}_{2}$.

In Fig. 8, we show a $7 \times 7$ elementary cell corresponding to an electrophilic 1,2 addition of $\mathrm{Br}_{2}$ to a $\mathrm{C}=\mathrm{C}$ bond near a line defect. This corresponds to two $\mathrm{Br}$ atoms added to $\mathrm{C}$ atom 4 and its neighbouring $\mathrm{C}$, at the line defect of Fig. 6. Fig. 8 can also be seen as a periodic analogue to the cluster model of Fig. 7(b).

In the figure, besides the elementary cell, NEXAFS spectra are shown for the same $\mathrm{C}$ atoms 1-8 as in Fig. 6, now for the brominated case (red), together with the intact surface (blue, dashed) and the Br-free line defect (black). We first of all note that the bromination leads to only small additional changes of
NEXAFS spectra for $\mathrm{C}$ atoms 1 and 2, beyond the Br-free case of Fig. 6 with a simple line-defect. This was to be expected due to the large distance of $\mathrm{C} 1$ and $\mathrm{C} 2$ from the defect. Similar moderate changes are found for $\mathrm{C}$ atoms $\mathrm{C} 3, \mathrm{C} 5, \mathrm{C} 6, \mathrm{C} 7$ and $\mathrm{C} 8$, which are also not in direct contact with a $\mathrm{Br}$ atom. The biggest variations are observed for atom $\mathrm{C} 4$, which is connected to a $\mathrm{Br}$ atom and which rehybridizes from $\mathrm{sp}^{2}$ to $\mathrm{sp}^{3}$ : the $\mathrm{C} 1 \mathrm{~s} \rightarrow \pi^{*}$ resonance at around $285.4 \mathrm{eV}$ broadens towards the "fingerprint" region and looses intensity. The $\mathrm{C} 1 \mathrm{~s} \rightarrow \sigma^{*}$ resonance shifts from about 292 to about $291 \mathrm{eV}$, gains intensity and broadens also towards the "fingerprint" region. Compared to defect-free graphene, for $\mathrm{C} 4$ both C 1s $\rightarrow \pi^{*}$ and C 1s $\rightarrow \sigma^{*}$ resonances loose intensity and are broadened/shifted towards the "fingerprint" region. This is in partial agreement with experiment, where upon bromination a decrease and possible splitting, of the $\mathrm{C} 1 \mathrm{~s} \rightarrow \pi^{*}$ transition was found, as well as increased intensity in the fingerprint region, vide supra. It should be noted that the overall effects due to physical defects on NEXAFS spectra dominate over additional effects of bromination, at least for the low-coverage model of Fig. 8.

In Fig. 9 we consider as a further model a $7 \times 7$ elementary cell corresponding to a radical addition of a single $\mathrm{Br}$ atom to $\mathrm{C}$ atom 6 of Fig. 6. Equivalently, Fig. 9 is the periodic analogue of cluster model Fig. 7(c). In this case, $\mathrm{C}$ atom 6 attached to $\mathrm{Br}$ remains $\mathrm{sp}^{2}$-hybridized, since $\mathrm{Br}$ formally replaces an $\mathrm{H}$ atom of Fig. 6. Again we find that bromination has a minor impact on NEXAFS spectra, compared to the situation where "only" a physical line defect was present. By far the biggest additional changes are for atom $\mathrm{C} 6$ as expected: here the $\pi^{*}$ feature increases its intensity and broadens, while the resonance in the middle of the "fingerprint" region vanishes. The $\mathrm{C} 1 \mathrm{~s} \rightarrow \sigma^{*}$ signal changes not by much. Compared to the defect-free surface, the $\pi^{*}$ resonance grows slightly, the C $1 \mathrm{~s} \rightarrow \sigma^{*}$ intensity decreases and the "fingerprint" region in between is hardly affected. These observations are in less good agreement with experiment if taken seriously, i.e., a partial rehybridization of $\mathrm{C}$ atoms to $\mathrm{sp}^{3}$ upon bromination seems to better fulfill boundary conditions imposed by experimental findings.

\section{Summary and conclusions}

In summary, we have shown that both physical and chemical modifications of graphene surfaces will have an influence on their C K-edge NEXAFS spectra. In particular, the following observations were made.

- Physical as well as chemical defects can lead to the rehybridization of $\mathrm{C}$ atoms in the graphene layer, from $\mathrm{sp}^{2}$ to $\mathrm{sp}^{3}$. In our examples above, this was the case for physical defect $\mathrm{V}_{1}(5,9)$ (Fig. 4) and the chemical defect in Fig. 8. Locally, the $\pi$ system is destroyed and as a consequence, the C $1 \mathrm{~s} \rightarrow \pi^{*}$ resonance (at $285.4 \mathrm{eV}$ ) is diminished.

- Also for other defects, even without $\mathrm{C}$ rehybridization to $\mathrm{sp}^{3}$, often reduced C 1s $\rightarrow \pi^{*}$ intensities are found. Further, C $1 \mathrm{~s} \rightarrow \sigma^{*}$ resonances (at around $298 \mathrm{eV}$ ) are frequently diminished. These effects may have to do with the fact that a defect can lead to a local shift of $\mathrm{C} 1 \mathrm{~s} \rightarrow \pi^{*}$ and $\rightarrow \sigma^{*}$ excitation energies. 

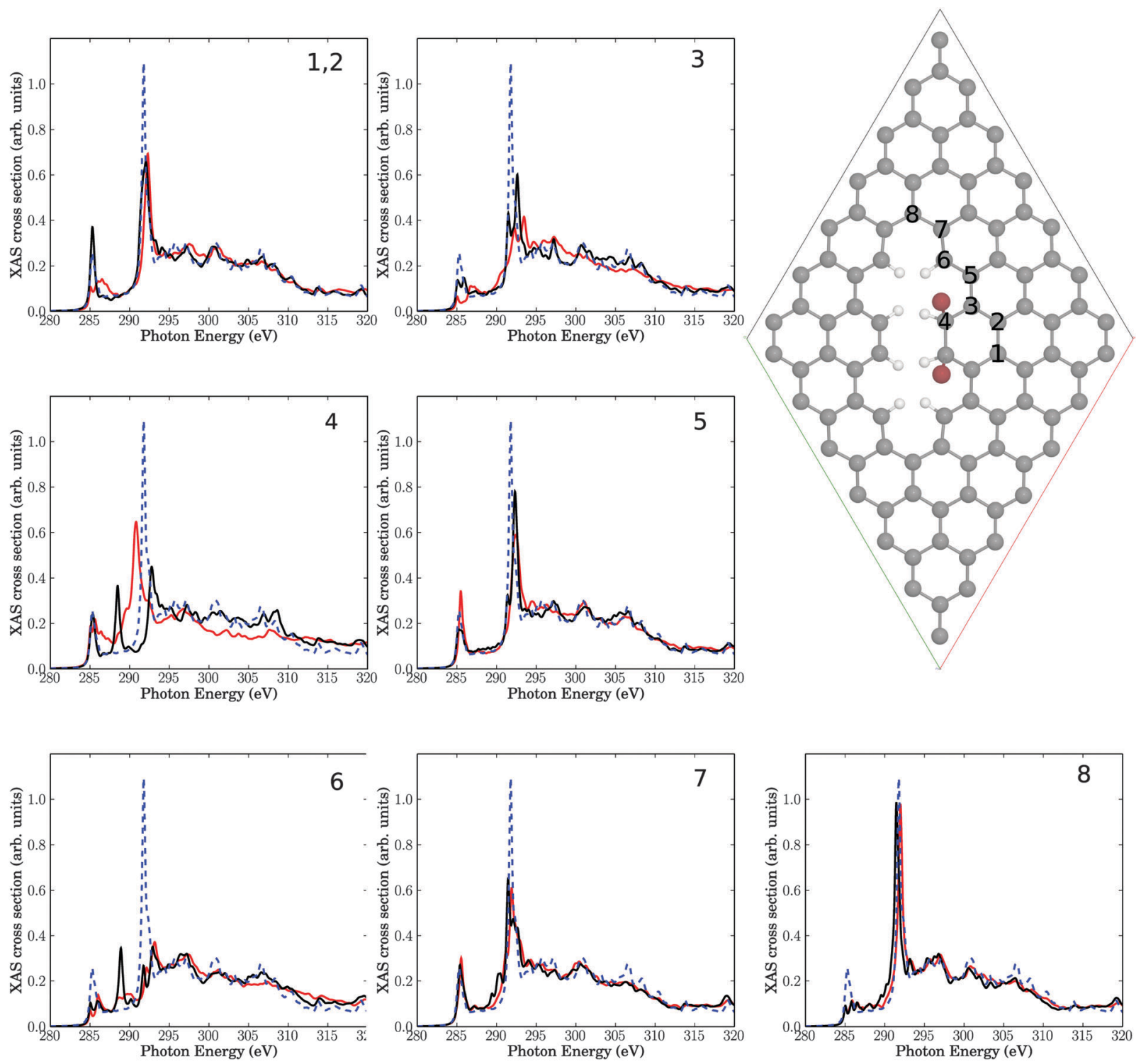

Fig. 8 Upper right: elementary cell for a situation with two $\mathrm{Br}$ atoms added to neighbouring $\mathrm{C}$ atoms $\left(\mathrm{sp}^{3}\right)$ near a line defect. The seven insets show NEXAFS spectra corresponding to core-hole creation in atoms 1-8, located close to the defect (same atom numbering as in Br-free Fig. 6). Full, red lines: computed NEXAFS spectra for brominated species, dashed, blue lines: the theoretical spectrum of unperturbed graphene for comparison. The black lines correspond to spectra for the Br-free line defect of Fig. 6.

- Often, defects cause additional resonances in the "fingerprint" region between the original $\pi^{*}$ and $\sigma^{*}$ resonances. In some cases, this arises from shifts of the $\pi^{*}$ states upward, or loss and shift of $\pi^{*}$ intensity by formation of $\mathrm{sp}^{3}$-hybridized $\mathrm{C}$ atoms. In most cases studied in this work, the new features in the "fingerprint" region are due to shifted and splitted $\sigma^{*}$ states: the three-fold symmetry around particular $\mathrm{C}$ atoms is broken, leading to splitted $\sigma^{*}$ levels with shifted energies, often to lower energies due to elongated $\mathrm{C}-\mathrm{C}$ bonds.

- The effects of defects on NEXAFS spectra are local, but not fully localized to $\mathrm{C}$ atoms in the center of a defect or to their nearest neighbours.

- The additional effects of chemical modification (bromination) on top of physical perturbations on NEXAFS spectra, are comparatively small. For the cases studied, physical defects dominate over chemical defects. It should be noted, however, that in both examples (Fig. 8 and 9), the $\mathrm{Br}$ load was low: two $\mathrm{Br}$ atoms per $94 \mathrm{C}$ atoms in Fig. 8, and one $\mathrm{Br}$ atom per $94 \mathrm{C}$ atoms for Fig. 9.

- In case of chemical modification, brominated species which otherwise induce no physical defects are expected to play a role at low $\mathrm{Br}$ concentrations only. At higher $\mathrm{Br}$ load, chemical and physical defects go hand in hand. In particular C$\mathrm{Br}$ bonds with $\mathrm{sp}^{3} \mathrm{C}$ atoms seem to form. At least, this will better account for measured NEXAFS spectra compared to those due to $\mathrm{C}-\mathrm{Br}$ bonds with $\mathrm{sp}^{2} \mathrm{C}$ atoms.

The trends observed by our theoretical models are consistent with experimental observations. ${ }^{6}$ It must also be clearly said, however, that a detailed, quantitative understanding of the experimental data is hardly possible at the moment. This would require a more detailed knowledge on the concentration 

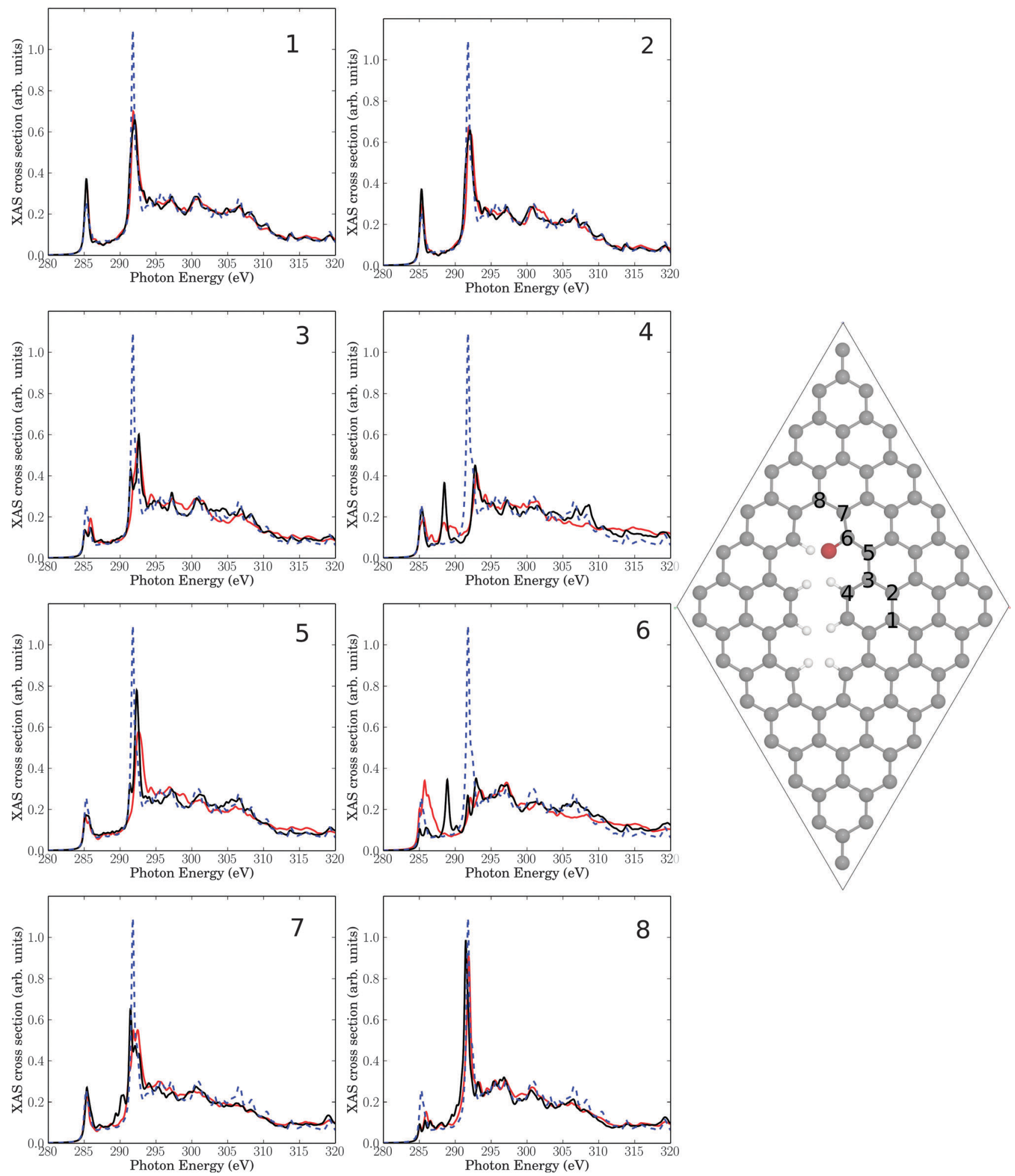

Fig. 9 Right: elementary cell for a single $\mathrm{Br}$ atom added to a $\mathrm{C}$ atom $\left(\mathrm{sp}^{2}\right)$ near a line defect. The eight insets show NEXAFS spectra for core-hole creation in atoms 1-8 close to the defect (same atom numbering as in Br-free Fig. 6). Full, red lines: computed NEXAFS spectra for brominated species, dashed, blue lines: the theoretical spectrum of unperturbed graphene for comparison. The black lines correspond to spectra for the Br-free line defect of Fig. 6 .

and statistical distribution of intact and defective carbon atoms, and a costly averaging over all non-equivalent $\mathrm{C}$ atoms. The selection of model systems studied here is somewhat arbitrary and certainly incomplete, albeit based to a good extent on stability criteria. The theoretical treatment, nevertheless, supports the experimental finding of NEXAFS as being a sensitive tool for defective graphenes. In addition, it offers a possible microscopic interpretation for experimental data. 


\section{Acknowledgements}

Thanks are due to the BAM Presidium for financial support through Project Ideen_2012_59 as part of the BAM MIS Program. Fruitful discussions with D. Kröner (University of Potsdam) are gratefully acknowledged.

\section{References}

1 K. S. Novoselov, A. K. Geim, S. V. Morozov, D. Jiang, Y. Zhang, S. V. Dubonos, I. V. Grigorieva and A. A. Firsov, Science, 2004, 306, 666.

2 A. H. Castro Neto, F. Guinea, N. M. R. Peres, K. S. Novoselov and A. K. Geim, Rev. Mod. Phys., 2009, 81, 109.

3 A. K. Geim, Science, 2009, 324, 1530.

4 H. Raza, Graphene Nanoelectronics: Metrology, Synthesis, Properties and Applications, Springer, 2012.

5 V. Georgakilas, M. Otyepka, A. B. Bourlinos, V. Chandra, N. Kim, K. C. Kemp, P. Hobza, R. Zboril and K. S. Kim, Chem. Rev., 2012, 112, 6156.

6 A. Lippitz, J. F. Friedrich and W. E. Unger, Surf. Sci., 2013, 611, L1.

7 J. Stöhr, NEXAFS Spectroscopy, Springer Series in Surface Sciences, Springer, 2003.

8 P. E. Batson, Phys. Rev. B: Condens. Matter Mater. Phys., 1993, 48, 2608.

9 F. Banhart, J. Kotakoski and A. V. Krasheninnikov, ACS Nano, 2011, 5, 26.

10 V. A. Coleman, R. Knut, O. Karis, H. Grennberg, U. Jansson, R. Quinlan, B. C. Holloway, B. Sanyal and O. Eriksson, J. Phys. D: Appl. Phys., 2008, 41, 062001.

11 T. Schiros, D. Nordlund, L. Plov, D. Prezzi, L. Zhao, K. S. Kim, U. Wurstbauer, C. Gutirrez, D. Delongchamp, C. Jaye, D. Fischer, H. Ogasawara, L. G. M. Pettersson, D. R. Reichman, P. Kim, M. S. Hybertsen and A. N. Pasupathy, Nano Lett., 2012, 12, 4025.

12 A. V. Okotrub, N. F. Yudanov, I. P. Asanov, D. V. Vyalikh and L. G. Bulusheva, ACS Nano, 2013, 7, 65.

13 W. Hua, B. Gao, S. Li, H. Ågren and Y. Luo, Phys. Rev. B: Condens. Matter Mater. Phys., 2010, 82, 155433.

14 R. Hamdan, A. F. Kemper, C. Cao and H. P. Cheng, J. Chem. Phys., 2013, 138, 164702.

15 A. Yaya, C. P. Ewels, I. Suarez-Martinez, P. Wagner, S. Lefrant, A. Okotrub, L. Bulusheva and P. R. Briddon, Phys. Rev. B: Condens. Matter Mater. Phys., 2011, 83, 045411.

16 A. N. Rudenko, F. J. Keil, M. I. Katsnelson and A. I. Lichtenstein, Phys. Rev. B: Condens. Matter Mater. Phys., 2010, 82, 035427.

17 W. Kohn and L. J. Sham, Phys. Rev., 1965, 140, A1133.

18 P. Giannozzi, S. Baroni, N. Bonini, M. Calandra, R. Car, C. Cavazzoni, D. Ceresoli, G. L. Chiarotti, M. Cococcioni, I. Dabo, A. D. Corso, S. d. Gironcoli, S. Fabris, G. Fratesi, R. Gebauer, U. Gerstmann, C. Gougoussis, A. Kokalj, M. Lazzeri, L. Martin-Samos, N. Marzari, F. Mauri, R. Mazzarello, S. Paolini, A. Pasquarello, L. Paulatto, C. Sbraccia, S. Scandolo, G. Sclauzero, A. P. Seitsonen, A. Smogunov, P. Umari and R. M. Wentzcovitch, J. Phys.: Condens. Matter, 2009, 21, 395502.
19 J. P. Perdew, K. Burke and M. Ernzerhof, Phys. Rev. Lett., 1996, 77, 3865.

20 N. Troullier and J. L. Martins, Phys. Rev. B: Condens. Matter Mater. Phys., 1991, 43, 1993.

21 M. Taillefumier, D. Cabaret, A.-M. Flank and F. Mauri, Phys. Rev. B: Condens. Matter Mater. Phys., 2002, 66, 195107.

22 H. J. Monkhorst and J. D. Pack, Phys. Rev. B: Solid State, 1976, 13, 5188.

23 C. Gougoussis, M. Calandra, A. P. Seitsonen and F. Mauri, Phys. Rev. B: Condens. Matter Mater. Phys., 2009, 80, 075102.

24 P. E. Blöchl, Phys. Rev. B: Condens. Matter Mater. Phys., 1994, 50, 17953.

25 W. Olovsson, I. Tanaka, T. Mizoguchi, G. Radtke, P. Puschnig and C. Ambrosch-Draxl, Phys. Rev. B: Condens. Matter Mater. Phys., 2011, 83, 195206.

26 L. Triguero, O. Plashkevych, L. Pettersson and H. Ågren, J. Electron Spectrosc. Relat. Phenom., 1999, 104, 195.

27 K. Hermann and L. G. M. Pettersson, M. E. Casida, C. Daul, A. Goursot, A. Koester, E. Proynov, A. St-Amant, D. R. Salahub, V. Carravetta, H. Duarte, C. Friedrich, N. Godbout, J. Guan, C. Jamorski, M. Leboeuf, M. Leetmaa, M. Nyberg, S. Patchkovskii, L. Pedocchi, F. Sim, L. Triguero and A. Vela, Be-deMon version 3.1, 2011.

28 M. J. Frisch, G. W. Trucks, H. B. Schlegel, G. E. Scuseria, M. A. Robb, J. R. Cheeseman, G. Scalmani, V. Barone, B. Mennucci, G. A. Petersson, H. Nakatsuji, M. Caricato, X. Li, H. P. Hratchian, A. F. Izmaylov, J. Bloino, G. Zheng, J. L. Sonnenberg, M. Hada, M. Ehara, K. Toyota, R. Fukuda, J. Hasegawa, M. Ishida, T. Nakajima, Y. Honda, O. Kitao, H. Nakai, T. Vreven, J. A. Montgomery, Jr., J. E. Peralta, F. Ogliaro, M. Bearpark, J. J. Heyd, E. Brothers, K. N. Kudin, V. N. Staroverov, R. Kobayashi, J. Normand, K. Raghavachari, A. Rendell, J. C. Burant, S. S. Iyengar, J. Tomasi, M. Cossi, N. Rega, J. M. Millam, M. Klene, J. E. Knox, J. B. Cross, V. Bakken, C. Adamo, J. Jaramillo, R. Gomperts, R. E. Stratmann, O. Yazyev, A. J. Austin, R. Cammi, C. Pomelli, J. W. Ochterski, R. L. Martin, K. Morokuma, V. G. Zakrzewski, G. A. Voth, P. Salvador, J. J. Dannenberg, S. Dapprich, A. D. Daniels, Farkas, J. B. Foresman, J. V. Ortiz, J. Cioslowski and D. J. Fox, Gaussian 09 Revision D.01, Gaussian Inc., Wallingford, CT, 2009.

29 S. Grimme, J. Antony, S. Ehrlich and H. Krieg, J. Chem. Phys., 2010, 132, 154104.

30 R. Krishnan, J. S. Binkley, R. Seeger and J. A. Pople, J. Chem. Phys., 1980, 72, 650.

31 Y. Ahmad, M. Dubois, K. Gurin, A. Hamwi, Z. Fawal, A. P. Kharitonov, A. V. Generalov, A. Y. Klyushin, K. A. Simonov, N. A. Vinogradov, I. A. Zhdanov, A. B. Preobrajenski and A. S. Vinogradov, J. Phys. Chem. C, 2013, 117, 13564.

32 J. Stöhr, F. Sette and A. L. Johnson, Phys. Rev. Lett., 1984, 53, 1684.

33 A. Krasheninnikov and R. Nieminen, Theor. Chem. Acc., 2011, 129, 625.

34 U. Singh and P. Kollman, J. Comput. Phys., 1984, 5, 129.

35 M. Ernzerhof and G. E. Scuseria, J. Chem. Phys., 1999, 110, 5029. 